insert (off-label use). Worryingly, there is little or no scientific evidence in support of the majority of off-label drug prescriptions. The authors call for mandatory postapproval surveillance to identify non-evidence-based prescribing practices: off-label prescribing can fast-track clinical advances in treatment, but might also jeopardize patient safety or result in unnecessary or ineffective treatment.

The team obtained data from the 2001 National Disease and Therapeutic Index (NDTI), which includes prescription data supplied at quarterly intervals by 3,500 randomly sampled US office-based physicians. They analyzed 160 medications (the 100 most frequently prescribed drugs, plus another 60 randomly selected medications from among those ranked by the NDTI). Their sample included $56 \%$ of all drug prescriptions for 2001.

Off-label prescriptions accounted for $21 \%$ of the sample. Almost half of prescriptions for anticonvulsants, and for cardiac medications other than hypertensive and lipid medications, were off label. When specific medications were analyzed, $81 \%$ of prescriptions for amitriptyline hydrochloride and $83 \%$ of prescriptions for gabapentin were off label. The authors note that drug manufacturers' marketing activities have been a matter for concern-WarnerLambert were prosecuted for promoting offlabel uses of gabapentin-and ask whether policy makers should allow off-label drug use without regulatory scrutiny, on the basis of scientific evidence; or whether these decisions should be left to market forces.

Original article Radley DC et al. (2006) Off-label prescribing among office-based physicians. Arch Intern Med 166: $1021-1026$

\section{The role of frozen-section analysis in nerve-sparing radical prostatectomy}

In men scheduled to undergo nerve-sparing radical prostatectomy, intraoperative findings of palpable subcapsular tumor usually indicate that the ipsilateral neurovascular bundle should be resected. Eichelberg et al. used intraoperative frozen-section analysis to assess such tumors, and found that the ipsilateral neurovascular bundle can be spared in almost half of patients with palpable tumors, without sacrificing oncologic efficacy.
A single surgeon performed 608 consecutive nerve-sparing radical prostatectomies, during which 83 palpable tumors were detected (13.7\%). Intraoperative frozen-section analysis revealed carcinoma in 77 of $83(93 \%)$ tumors. In 48 (58\%) tumors, frozen sections showed no capsular involvement, and the ipsilateral neurovascular bundle was spared. Final histopathology revealed positive surgical margins in 8 of 48 tumors: in 5 cases, the ipsilateral side had positive margins (equivalent to a falsenegative rate of $6 \%$ for detecting capsular involvement), and in 3 patients, the contralateral margins were positive. Interestingly, in 30 of 35 $(86 \%)$ cases in which the neurovascular bundle was resected because frozen-section analysis showed tumor contact with the capsule, final histopathology showed no tumor in the additionally resected tissues. Overall, positivemargin rates were similar in palpable versus nonpalpable tumors (15.7\% versus $14.3 \%$ ).

Although intraoperative frozen-section analysis would leave some patients with positive surgical margins, for some men, the improved continence and potency achievable with nervesparing surgery might outweigh the increased risk of recurrence associated with positive margins. These risks and benefits should be adequately discussed with the patient before surgery, say the authors.

Original article Eichelberg C et al. (2006) Frozen section for the management of intraoperatively detected palpable tumor lesions during nerve-sparing scheduled radical prostatectomy. Eur Urol 49: 1011-1018

\section{Is retroperitoneal laparoscopic heminephrectomy beneficial in infants?}

A single-center, retrospective analysis of 22 retroperitoneal laparoscopic heminephrectomies has revealed that serious complications can develop in infants aged $<1$ year who undergo this procedure, even some time after surgery. Wallis et al. suggest that physicians' enthusiasm for the low morbidity and good cosmesis of laparoscopic surgery should be tempered with caution in this age-group.

The authors treated 18 boys and 5 girls (mean age 5 years, range 4 months to 18 years). Four patients required conversion to open surgery because of inadequate exposure of the kidney; three had minor postoperative urine leaks that 